\title{
Deep Learning Driven Wireless Communications and Mobile Computing
}

\author{
Huaming Wu $\left(\mathbb{D},{ }^{1}\right.$ Zhu Han, ${ }^{2}$ Katinka Wolter, ${ }^{3}$ Yubin Zhao, ${ }^{4}$ and Haneul Ko ${ }^{5}$ \\ ${ }^{1}$ Tianjin University, Tianjin 300072, China \\ ${ }^{2}$ University of Houston, Houston, TX 77004, USA \\ ${ }^{3}$ Freie Universität Berlin, Berlin 14195, Germany \\ ${ }^{4}$ Chinese Academy of Sciences, Shenzhen 518055, China \\ ${ }^{5}$ Korea University, Sejong 30019, Republic of Korea
}

Correspondence should be addressed to Huaming Wu; whming@tju.edu.cn

Received 28 March 2019; Accepted 28 March 2019; Published 28 April 2019

Copyright (C) 2019 Huaming Wu et al. This is an open access article distributed under the Creative Commons Attribution License, which permits unrestricted use, distribution, and reproduction in any medium, provided the original work is properly cited.

With the exploding amount of mobile traffic data and unprecedented demands of computing, executing the ever increasingly complex applications in resource-constrained mobile devices becomes more and more challenging. Future wireless communications will be very complex with heterogeneous radio access technologies, transmission links, and network slices. More intelligent technologies are required to address those complex scenarios and to adapt to dynamic mobile environments. Recently, deep learning has attracted much attention in the field of wireless communication and mobile computing. Deep learning driven algorithms and models can facilitate wireless network analysis and resource management, benefit in coping with the growth in volumes of communication and computation for emerging mobile applications. However, how to customize deep learning techniques for heterogeneous mobile environments is still under development. Learning algorithms in mobile wireless systems are immature and inefficient. In this special issue on deep learning driven wireless communication and mobile computing, we have accepted seven papers that include both theoretical contributions and practical research related to the new technologies, analysis, and applications with the help of artificial intelligence and deep learning.

One paper proposes a new fault detection mechanism based on support vector regression among sensor observations in wireless sensor networks according to the redundant information of meteorological elements collected by multisensors. The proposed algorithm reduces the communication to sensor nodes and further achieves a high detection accuracy and a low false alarm ratio, which are more suitable for fault detection in meteorological sensor networks, even when the failure rate is very high.

Another paper presents a survey on deep learning techniques in signal recognition. Classical methods, emerging machine learning, and deep leaning schemes are extended from modulation recognition to wireless technology recognition. This survey discusses the open problems, new challenges, and future development trends on signal recognition in practical applications, e.g., burst signal recognition and unknown signal recognition.

One of the papers addresses the traffic explosion problem in hierarchical wireless networks. It proposes a Double Deep Q-network (Double DQN) based edge caching strategy, in order to maximize unloading traffic and reduce pressure through D2D communication. The proposed cache replacement strategy is established using a Markov decision process (MDP) and a deep reinforcement learning framework.

Another paper presents an integrated method combining a deep neural network (DNN) with an improved K-Nearest Neighbor (KNN) algorithm to enhance the accuracy of indoor positioning. The DNN algorithm is first used to classify the WiFi RSSI Fingerprinting dataset, and then 
these possible locations are classified by the improved KNN algorithm to determine the final position.

Another paper aims to understand the intention of legal consultation of users with different language expressions and legal knowledge background. It combines deep neural network Bidirectional Long Short-Term Memory (Bi-LSTM) with pattern-oriented tensor decomposition to perform the classification and deep understanding of a users' legal consulting statements. Experiments show that the proposed method is more instructive and interpretable, and it also demonstrates faster convergence and higher accuracy than the traditional deep neural networks.

One of the papers addresses the issue of how to rank nodes from positive and negative views of social network data mining. It develops a random walk based method for signed networks that allows the walker to change between positive and negative while crossing from one node to another.

Another paper proposes a deep Convolutional Neural Network (CNN) assisted personalized recommendation framework for mobile wireless networks. It combines the mobile user's location trajectory sequence, user-shared images, and text information and recommends potential visiting locations to users. This is achieved by using the big data sampled as the user's social and mobile trajectory and processing it through the $\mathrm{CNN}$ network.

We have been impressed with the variety of topics submitted to this special issue and we hope the reader will enjoy the papers as much as we did. We also hope that the deep learning driven algorithms and models demonstrated in this special issue will be helpful to develop customized deep learning techniques for heterogeneous wireless network architectures, mobile applications, and mobile systems.

\title{
Conflicts of Interest
}

As guest editors, we have no conflicts of interest to the accepted papers.

\section{Acknowledgments}

We thank all authors for their submission to this special issue and we thank all reviewers for their high-quality feedback.

\author{
Huaming Wu \\ Zhu Han \\ Katinka Wolter \\ Yubin Zhao \\ Haneul Ko
}




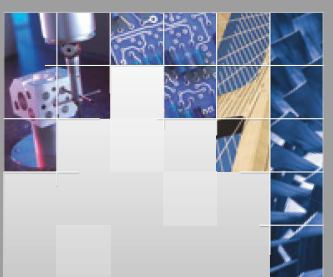

\section{Enfincering}
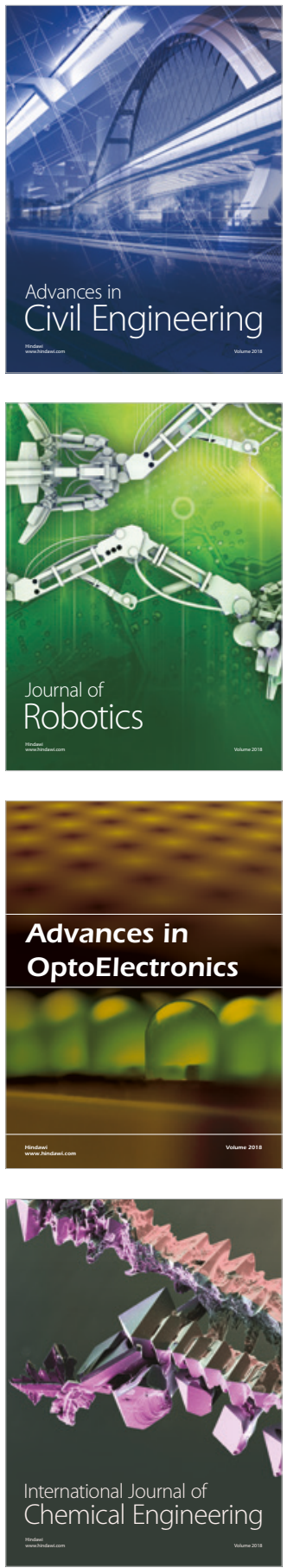

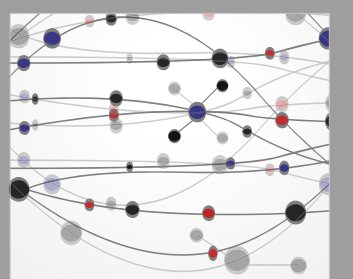

\section{Rotating \\ Machinery}

The Scientific World Journal

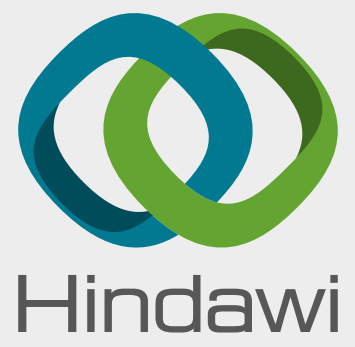

Submit your manuscripts at

www.hindawi.com
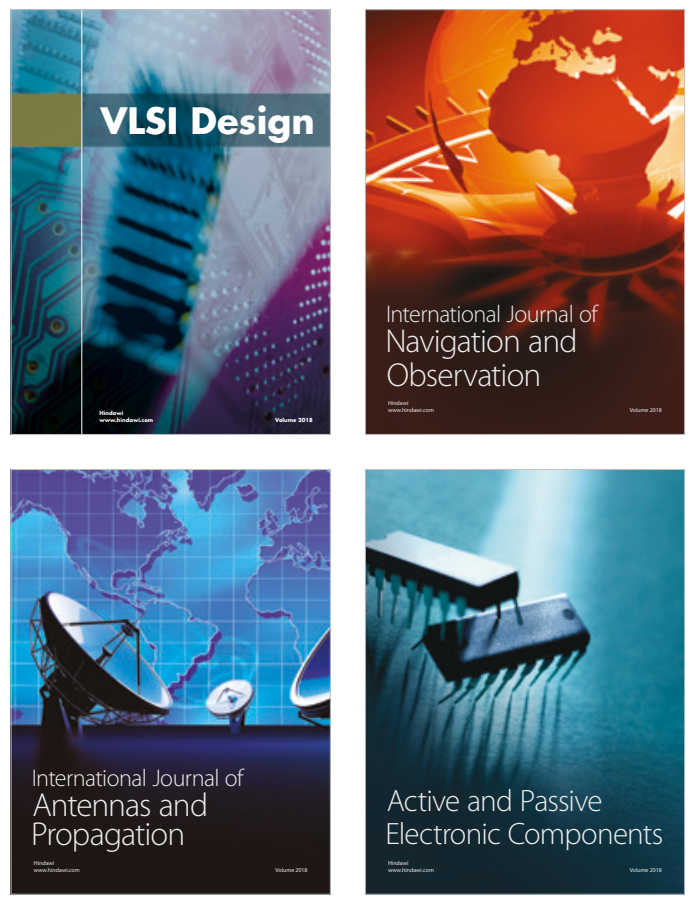
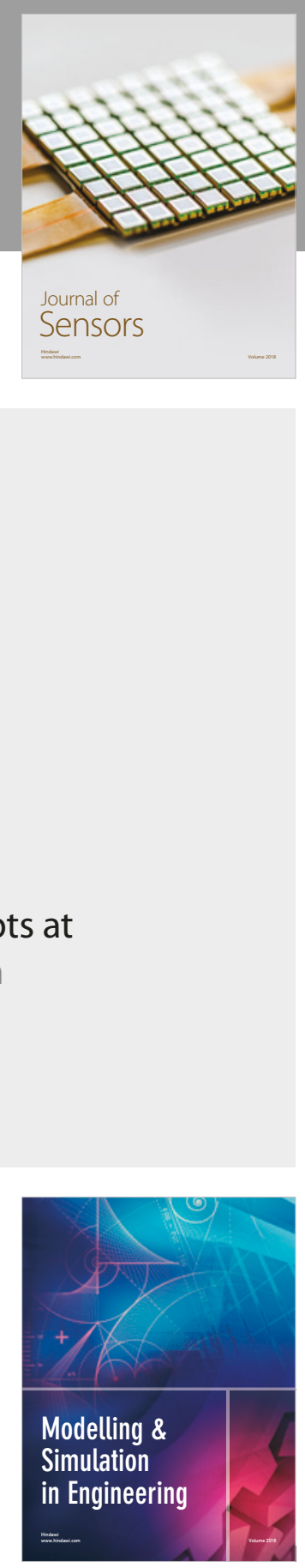

\section{Advances \\ Multimedia}
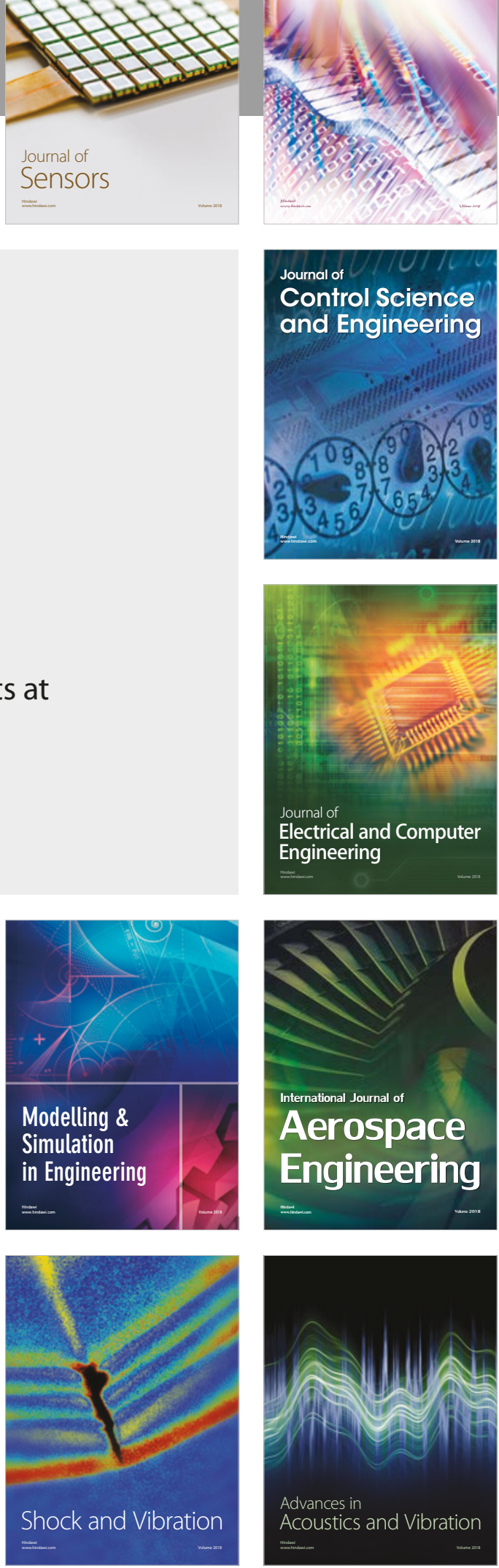